\title{
Research on Multi-user Growth Strategy of Pinduoduo Based on AARRR Model
}

\author{
Lin Zhang * \\ School of Economics and Management, Beijing Jiaotong University, Beijing, China \\ ${ }^{*}$ Corresponding author. Email: 292700307@qq.com
}

\begin{abstract}
Based on the rapid development of Internet technology, e-commerce platforms have risen rapidly. As a latecomer in the e-commerce industry, Pinduoduo has achieved explosive growth of users in a short period of time by virtue of the innovative business model of "social + e-commerce". And quickly achieve a platform breakthrough in the fiercely competitive e-commerce industry. At this stage, the e-commerce market has basically matured, and user growth is slowing down. How to recognize the current situation and formulate appropriate strategies to achieve further user stability and growth is an urgent problem for major e-commerce platforms.

In this context, based on the AARRR user conversion funnel model, this article takes Pinduoduo as the research object, discusses its user growth strategies at various stages, and proposes targeted improvement suggestions on this basis, with a view to contributing to Pinduoduo's sustainable development. Provide support and guarantee, and provide reference and reference for other current e-commerce platforms and even Internet companies to achieve further user growth.
\end{abstract}

Keywords: Pinduoduo, Social e-commerce, User growth, AARRR model.

\section{RESEARCH BACKGROUND AND MEANING}

In recent years, based on the rapid development of Internet technology, e-commerce platforms have rapidly emerged, and have gradually subverted people's traditional shopping concepts. Looking at the current ecommerce market, Taobao, JD, and Pinduoduo, as the three giants, have formed a tripartite situation. Pinduoduo was established in 2015. As a latecomer to the ecommerce industry, it has achieved a platform breakthrough in a short period of time by relying on the e-commerce model of "social + e-commerce".

At present, the e-commerce market is fiercely competitive and becoming mature and stable, the platform represented by Pinduoduo already has a certain number of user bases. Compared with the initial establishment of the platform, the focus is on acquiring users. At this stage, how to obtain more efficient users, how to keep stable users and achieve continuous user growth will be a greater challenge.

In this context, based on the AARRR user conversion funnel model, this article takes Pinduoduo as the research object, discusses its user growth strategies at various stages, and proposes targeted improvement suggestions on this basis, with a view to contributing to Pinduoduo's sustainable development provide support and guarantee, and provide reference for other current e-commerce platforms and even Internet companies to achieve further user growth.

\section{AARRR MODEL}

The concept of user growth originated in the Silicon Valley of the United States. User growth is the process of turning "passengers" into users [1]. The core of user growth is to help start-ups acquire a large number of users in a short period of time at a very low or even zero cost. [2]. With the continuous exploration and practice of global growth hackers, some efficient user growth frameworks have gradually formed in the market, which can make user growth more effective. Among them, the AARRR model is currently the most frequently mentioned and used framework in user growth.

AARRR is a set of indicators proposed by Dave McClure, the founder of 500 Startups, a well-known entrepreneurial incubation center in Silicon Valley [3], which specifically refers to acquisition, activation, retention, revenue, refer five stages [4]. Figure 1 shows the model. 


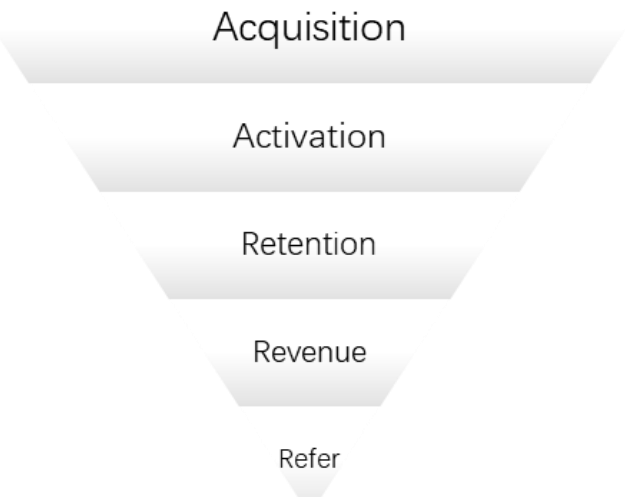

Figure 1 AARRR Model

This model can help companies think about the issues in the product development process in the table 1 :

Table 1. Enterprise Decision and AARRR Model

\begin{tabular}{|c|l|}
\hline Stage & Questions \\
\hline Acquisition & $\begin{array}{l}\text { - What channel do users come from? } \\
\cdot \text { How do users come? } \\
\cdot \text { How to let users discover products } \\
\text { or services and start trying them? }\end{array}$ \\
\hline Activation & $\begin{array}{l}\cdot \text { How to make users perceive the } \\
\text { value of products or services? } \\
\cdot \text { How to let users use the product } \\
\text { more frequently? }\end{array}$ \\
\hline Retention & $\begin{array}{l}\cdot \text { How to attract customers to return } \\
\text { visits? } \\
\cdot \text { How to improve the relationship } \\
\text { with customers so that they are } \\
\text { willing to exist for a long time? }\end{array}$ \\
\hline Revenue & $\begin{array}{l}\cdot \text { How can products or services } \\
\text { increase revenue through users? }\end{array}$ \\
\hline Refer & $\begin{array}{l}\cdot \text { Are users satisfied with the product } \\
\text { or service? } \\
\cdot \text { How to let users voluntarily share } \\
\text { the product and spread it to more } \\
\text { people? }\end{array}$ \\
\hline
\end{tabular}

\subsection{Acquisition}

As the first step in AARRR, customer acquisition is the beginning of all stories [5]. User acquisition refers to the process by which potential users come into contact with the product for the first time [6] and finally turn into product users. At the same time, it should be noted that for user acquisition, the most basic and important principle is CLV>CAC: that is, when the customer lifetime value is higher than the customer acquisition cost, user growth is sustainable, and it makes sense to acquire new users.

If you want to acquire users, you must first locate the target market based on the corporate strategy, and secondly, select the appropriate marketing channels for the target market. There are many channels for acquiring new users, including paid channels, organic channels, and other channels. Various types of channels have their own advantages and disadvantages. Companies should consider their own situation and product characteristics, comprehensively considering the potential user volume of the channel, user value, channel cost and other factors, and then select the appropriate channel to promote the product and acquire users.

\subsection{Activation}

User activation means that after the user is acquired, through product portfolio and event planning, to guide users to complete certain "designated actions", intensify the experience in the process of consumer products, so that they become long-term active loyal users [7]. In this process, users will gradually discover the value of the product and repeatedly use the product's functions, thereby realizing the conversion of user traffic. New user activation is a necessary intermediate link between new user acquisition and old user participation. At this stage, if the user perceives the value of the product and has a good user experience, they will continue to return visits, and eventually gradually transform into active loyal customers of the product and recommend the product to others; otherwise, the user may abandon the product and turn to use other products.

\subsection{Retention}

User retention means that after a user starts to use the product, after a period of time, they still continue to use the product. Retention is not just the next day retention in a narrow sense, but all the indicators that can be helpful to user retention [8]. User retention rate refers to the proportion of users who still launch the application after a period of time out of the number of new users in a certain statistical period, and is an important indicator of user activity. In the Internet industry, the cost of maintaining old users is much lower than the cost of attracting new users. Therefore, we must increase the frequency of user use as much as possible, reduce user churn, and increase user retention, so as to maintain the value of the product and extend the life cycle of the user at this stage, it is necessary to maintain interaction with users and enhance user stickiness by continuously optimizing products and improving services.

\subsection{Revenue}

Revenue is user monetization, and Internet industry monetization refers to traffic monetization, that is, users are willing to pay for your products, services, or content. In the Internet industry, users $=$ traffic $=$ money. This means that if you want to achieve revenue, you must have enough traffic, and if you want to have enough traffic, you must have a huge user base. Therefore, the first three links in the AARRR model-user acquisition, user activation, user retention is the basis for realizing 
revenue, and only when the user base reaches a certain scale, revenue is possible. At this stage, pay attention to providing paid products, services or values that meet user expectations, and avoid excessive commercialization that affects customer experience. In addition to charging users, companies can also make profits through advertising, games, traffic diversion, e-commerce, business sharing, value-added services and other methods.

\subsection{Refer}

User refer refers to a way to acquire new users by using the social influence of existing users [9]. It is a very low-cost and highly continuous promotion method based on customer relationships. Acquaintance refer are often more convincing than high authority and advertisements. If a company has successful user refer, it will bring about an astonishing explosive growth of users. Enterprises can use various incentives and preferential policies to induce customers to actively carry out information dissemination through their own channels by providing content information for dissemination, so as to achieve the purpose of publicity and promotion.

From user acquisition to user refer, the entire AARRR funnel model constitutes a closed loop of a complete user life cycle. As the user growth phase continues to deepen, the number of users who leak into the next phase is getting less and less, and the users who are finally left are users who have long-term value to the enterprise. The AARRR model connects the content of user conversion, operation stage and index system, forming a complete set of user analysis methods and operation system, thus playing a significant role in marketing and optimizing operations [10].

\section{OVERVIEW OF PINDUODUO}

Pinduoduo was established in September 2015. It is a third-party social e-commerce platform targeting C2M group shopping. As the pioneer of new e-commerce, it is committed to combining entertainment and social networking with e-commerce operations, allowing more users to enjoy a new shared shopping. Adhering to the operating philosophy of "good goods are not expensive", it is committed to serving the vast majority of ordinary consumers in China, attracting merchants through preferential policies, and providing tens of billions of subsidies to enable consumers to purchase discounted famous brand products, agricultural products of origin, factory products and new brand products. In a short period of time, it attracted a large number of merchants and user traffic, and achieved rapid development.

\section{PINDUODUO USER GROWTH STRATEGY}

\subsection{Current Situation of Pinduoduo's User Growth Strategy}

The AARRR model represents the five stages of the user life cycle. With the deepening of user growth, Pinduoduo adopts different strategies in the five stages.

User acquisition is the beginning of user growth. For e-commerce platforms, obtaining traffic is very important and is the basis for the platform's survival. Firstly, Pinduoduo accurately locates the target market and uses the third and fourth long-tail markets to acquire users. Secondly it create "Pinduoduo, Pinduoduo, use more, save more..." commercial song. Thirdly, it titles popular variety shows such as Zhejiang Satellite TV's "New Chinese Sing", Hunan Satellite TV's "Happy Camp" so as to carry out advertising campaigns to reach potential users. Finally, Pinduoduo motivates users to share links to Moments to achieve viral spread through order discounts and assistance in bargaining.

User activation is the second stage of the user growth model. After acquiring users, if they are not activated in time, user acquisition will be meaningless, and it will also face the risk of user loss. Pinduoduo achieves user activation through key actions such as clicking sharing links, group buying, bargaining for free, and participating in new user discount activities. When a user enters Pinduoduo with one of the above key behaviours, it will form a sense of identity and emotional link with Pinduoduo due to Pinduoduo's low price and preferential characteristics, which will further generate a desire to use APP.

User retention is the most important link in achieving user growth. Improving user retention requires companies to maintain good interaction with users and attract users to return visits repeatedly. In the process of marketing and promotion, accurately grasping the psychological drive of consumers and rationally using appropriate operating activities can effectively increase the user retention rate. Pinduoduo achieves user retention through WeChat official account push, background message bar reminders, screen opening gift package discounts and other gamified operations activities such as bargaining for free, assisting red envelope withdrawal, signing in and receiving red envelopes, and Duoduo Orchard.

Revenue is the basis for a company's long-term survival. Pinduoduo does not directly generate transactions with consumers, but serves as a trading platform for merchants and users to achieve a win-win situation for the three parties through merchants. Pinduoduo obtains transaction service income by charging merchants for technical service fees, and obtains marketing service income by means of 
monetization and store bidding ranking. In addition, in the fourth quarter of 2020, Pinduoduo launched Duoduo's grocery shopping business, which is a self-operated business and is currently Pinduoduo's second largest source of revenue.

As the number of users continues to expand, user increments will gradually slow down, and making full use of old users to recruit new users is an important means to achieve user growth. The rapid growth in the number of Pinduoduo users is also due to the sharing recommendations of old users. Pinduoduo achieves viral spread through friends grouping, bargaining assistance, red envelope withdrawal assistance, word-of-mouth recommendation, etc. The motivation for users to recommend products is:

(1) Buying goods at low prices: Old users invite their friends and relatives to join the group. On the one hand, they can enjoy preferential prices, and on the other hand, they can also help relatives and friends to save money. Since then, Pinduoduo has achieved brand promotion at a very low cost.

(2) Free access to goods and red envelopes: Inviting relatives and friends to bargain can help old users and new users get free goods, and inviting relatives and friends to help get large red envelopes for free cash out opportunities, and this activity needs to be constantly refreshed. Fission propagation has realized explosive growth of K-factor recommended by users.

\subsection{Suggestions for Pinduoduo's User Growth Strategy Optimization}

\subsubsection{Broaden Marketing Channels}

At this stage, marketing methods such as WeChat sharing and TV advertising have achieved the acquisition of most of the target user groups, and viral moments link sharing has caused some users to over-socialize, reduce user experience, and cause other users' disgusted. Pinduoduo should actively expand other channels to achieve further acquisition of potential users and lost users.

Carry out joint marketing. Pinduoduo can cooperate with other APPs to carry out joint membership marketing. Pinduoduo can cooperate with Tencent Video and become its affiliated member, relying on the resources of partners in different industries to obtain more user traffic introduction and achieve further user acquisition.

Create an IP image. Pinduoduo can combine its own positioning and philosophy to create distinctive IP, enhance the exposure of IP image, and use IP image to further deepen brand memory, thereby attracting users.

Enable celebrity endorsements. Compared with Tmall's choice of Yi Yang Qianxi as its spokesperson,
JD.com chose Li Xian and Yang $\mathrm{Zi}$ as its spokesperson, Pinduoduo's spokesperson is rarely known. Pinduoduo can try to choose celebrity endorsements whose own growth experience and personal image are consistent with the brand, so as to drive the fan economy with celebrity effects.

Try offline marketing. Pinduoduo can try to carry out offline marketing. Set up offline venues to hold events, build new consumption scenes through interaction and other methods, build an immersive consumption experience to enhance interaction with users, and attract more new users to join.

\subsubsection{Improve Product Quality}

In the early days of Pinduoduo's establishment, the low threshold for merchants to settle in led to the existence of counterfeit and inferior products. In addition, under the $\mathrm{C} 2 \mathrm{M}$ model, merchants purchase raw materials in large quantities, and some merchants use shoddy products in order to obtain more benefits. In addition, large-scale production of factories reduces operating costs and shortens time, making it difficult to guarantee the quality of goods. In the e-commerce industry, products are the foundation for maintaining platforms, merchants and customers. Only good products can maintain users. Pinduoduo should improve product quality, win user trust, and increase user loyalty.

Raise the barriers to entry. First, strictly check the business qualifications of the business before entering, and secondly, investigate the customer evaluation and credibility level of other platforms. Third, sign contracts related to credit management, clearly indicating the punishment system for counterfeit and inferior products in the course of business operations. Finally, in the course of business operations, merchants are required to make detailed disclosures of products and improve the supply mechanism.

Supervise and random inspections of commodities. Pinduoduo should design and develop software to check products by supervising product keywords, and at the same time, random inspections of products on the shelves of merchants from time to time. Severely punish merchants with unqualified products, reward merchants with outstanding performance, and achieve a clear distinction between rewards and punishments and concurrent rewards and punishments.

Invest in sourcing factories. In addition, Pinduoduo can directly invest in manufacturing plants. On the one hand, as an investor, it can deeply control the supply side and effectively supervise and manage the quality of goods directly at the source of supply. On the other hand, it can enjoy income dividends and obtain profit. 


\subsubsection{Improve Service Experience}

Improving product quality is to protect the rights and interests of consumers from the perspective of the supply side, while improving service experience is to protect the rights and interests of consumers from the demand side. The combination of the two can improve user satisfaction. Relevant data shows that the complaint resolution rate of Pinduoduo users is very low. Due to the particularity of Pinduoduo's low price, its refund and return are slightly different from other e-commerce platforms. Small profits but quick turnover is the basis for the profitability of major merchants. When the problem of refund and return occurs again, there will be no profit for the merchants. Therefore, this has also led to the problem that many merchants are not active in aftersales service. In addition, Pinduoduo lacks a strong and complete supervision system for merchants, resulting in uneven service attitudes among merchants. At present, consumers are relatively dissatisfied with Pinduoduo's after-sales service. Pinduoduo should promptly resolve user complaints and improve service experience.

Unified customer service training. Pinduoduo should conduct unified training for merchant customer service, and after the training is over, carry out simulation tests to verify the learning results of communication with customers, improve the level and quality of platform practitioners, and provide customers with trustworthy after-sales protection.

Resolve complaints in a timely manner. Pinduoduo should increase the number of customer service, formulate internal service rules, improve the service standards of the service department, resolve user complaints in a timely manner, appropriately adopt reasonable suggestions and feedback from customers, and severely punish businesses that frequently complain.

\subsubsection{Enriching Operational Activities}

Rich and targeted operational activities are essential for the retention and newness of the platform. Pinduoduo achieves user recruitment, activation and retention through a series of social gamification operations. However, related activities also have problems such as unclear interpretation of the rules, excessive links arousing disgust in the circle of friends, high cost of user input time, and false commodity prices. Pinduoduo's platform activity design is unreasonable. Problems such as raising prices then bargaining and unclear rules are deceptive and excessively consume the trust of users. Pinduoduo should rectify existing activities, develop and innovate activities, and improve the design to enhance user trust.

Improve the rules of activities. Firstly, place a price graph on the product page to ensure that users are clear about the recent price changes of the product, and it is forbidden to increase the price before bargaining for active products. Secondly, in activities such as "bargain for free" and "help red envelope withdrawal", clearly indicate the interpretation information of the relevant rules to effectively protect consumers' right to know.

Expand the type of activity. In addition to the existing activities such as bargaining, assistance, orchard, Pinduoduo can use its existing user base to carry out a series of special activities aimed at its main business of agricultural products. For example, Pinduoduo can carry out remote breeding activities and sell agricultural products in the form of remote gamification planting, so that users can truly see the growth process of agricultural products and increase user trust.

\subsubsection{Optimize Platform Experience}

Platform experience is also an important part of achieving user retention. A good platform experience is also the basis for realizing user active recommendation. In order to attract users to return visits, Pinduoduo often pushes various preferential activities and limited-time promotions or information requested by friends in the background from time to time, resulting in too frequent push of background messages. In addition, in terms of personal privacy and security, login and purchase can be realized by directly clicking on the link shared by the user. Although the cumbersome personal information filling and registration process is simplified, the security verification steps are missing, and there are security risks. Pinduoduo currently urgently needs to strengthen its background message push and user privacy and security.

Reduce the frequency of push. In its daily operations, Pinduoduo should moderately reduce the frequency of background message pushes, reduce user resistance, and adopt different push strategies for users' frequency of use. For example, users with a high frequency of use can push more products they are interested in based on their usual browsing history to attract them repurchase; for users with a low frequency of use, they can push more discounts and promotions to attract their return visits.

Strengthen information protection. With the rapid development of technology and the advent of the era of big data, information theft incidents emerge in endlessly. Pinduoduo should strengthen the attention to user privacy and security, optimize platform technology, strengthen secret protection mechanisms, reduce off-site links, and strengthen the authenticity of links. For new users, add personal information verification steps in the login link to strengthen the protection of user information and ensure that consumers complete the shopping experience in a safe network environment. 


\subsubsection{Upgrade Revenue System}

Revenue is the foundation of the company's survival. Pinduoduo's revenue sources have problems such as fewer revenue channels and a single structure. Pinduoduo should make full use of its existing user and traffic base to upgrade its revenue system.

Increase revenue channels. Pinduoduo can adjust its 10 billion subsidy "commission-free" strategy, give full play to the basic rights of the e-commerce platform, collect some commissions from participating merchants with 10 billion subsidies, and increase revenue channels.

Broaden the scope of business. First of all, Pinduoduo should vigorously develop its self-operated business "Buy Duoduo", and use this as a starting point to deeply intervene in logistics, customer service, after-sales and other service links to establish a more mature operating system. Secondly, with continuous development and growth, a single type of business has been unable to meet the needs of platform expansion. Pinduoduo should focus on diversified business layout, build an ecological flow system, and expand its business to ancillary service areas such as Internet finance, logistics and distribution, and business entertainment.

\section{CONCLUSION}

Based on the case analysis of Pinduoduo, this article summarizes its user growth strategy based on each link of the AARRR model, analyses the problems faced by its current user growth strategy, and gives targeted improvement measures, which fully illustrates the user conversion funnel model Guiding role in the process of enterprise user growth.

Although Pinduoduo has made certain achievements in user growth, it still has many shortcomings. Problems such as narrow marketing channels, low product quality, poor service experience, unclear activity rules, and poor platform experience have reduced user satisfaction, resulting in the loss of some users. In addition, the monotonous problem of revenue channels also makes it a big gap with Ali and JD.com. Pinduoduo should face up to its own development problems, use its existing advantages and overcome current weaknesses. By broadening marketing channels, improving product quality, improving service experience, enriching operational activities, optimizing platform experience, and upgrading revenue system, we optimize all stages of the AARRR user conversion funnel model to improve user satisfaction and achieve further user growth.

In today's increasingly competitive business environment, choosing a scientific and efficient user growth model combined with data analysis and technology drive to continuously improve user experience and achieve user growth is the wisest choice for major companies. In the future, I hope that more scholars can invest in the research of user growth models, provide reference and experience for other e-commerce platforms and even other Internet platforms, and promote the development of e-commerce and the Internet industry.

\section{REFERENCES}

[1] Zhang Yifan. How should Chinese-style user growth be done? in: Sales and Marketing (Marketing Edition), 2020, pp. 94-95.

[2] Li Yinghan, Research on Airbnb User Growth Strategy Based on AARRR Model, in: Beijing University of Posts and Telecommunications, 2018.

[3] Fan Bing, Growth Hacking: Cheats for User and Revenue Growth of Startup Companies, Electronic Industry Press, 2015.

[4] Liu Nian, Analysis of the operation strategy of each stage of the AARRR model, 2018, DOI: http://www.woshipm.com/operate/887464.html

[5] Gao Xiaolong, User Operation Practical Guide, China Machinery Industry Press, 2018.

[6] Sean Ellis, Morgan Brown, Growth hackers-how to achieve explosive growth at low cost, CITIC Press, 2018.

[7] Zong Ping, Analysis of Network Marketing of Catering Enterprises Based on AARRR Model, in: Journal of Sichuan Institute of Tourism, 2019, pp.40-43.

[8] Zhang Ziwei, User growth and operational combat, Electronic Industry Press, 2018.

[9] Tan Jing, New media marketing operation combat, People's Posts and Telecommunications Press, 2017.

[10] Xu Qianru, Research on user retention strategy of freight APP based on AARRR model, in: Electronic Commerce, 2019, pp. 66-67. 